by the establishment of a new and active cortical circulation, by which the diseased cells of the uriniferous tubules are regenerated and their functional activity increased. I look upon this view as entirely erroneous, and not supported by facts. I have never seen any such new formation of blood vessels in kidneys which have been decapsulated, quite the contrary, the new-formed fibrous tissue is usually found to be almost non-vascular when a few months old. When the capsule of the kidney is removed granulation tissue forms on the surface of the organ, and blends with the granulation tissue thrown out by the tissues of the parietes. At first the capillary circulation in this new cellular connective tissue is very active, but no new vessels of any size form, and within a comparatively short time it becomes converted into dense fibrous tissue.

Certainly Edebohls has rendered good service to the profession by the enthusiastic advocacy of surgical interference in certain forms of albuminuria, but I do not consider that his method of decapsulation does more good to the patient than relief of tension by simple incision, as advised by $\mathrm{Mr}$. Reginald Harrison in 1896 , and as practised by me in $1888 .{ }^{1}$ It is true that in the instance referred to the operation was undertaken in the belief that the patient was suffering from renal calculus, but the operation and its result taught me that by incising the kidney "relief of tension facilitates the renal circulation," it relieved pain and caused the albuminuria to cease. This lesson I have always remembered, and it has regulated my practice in such cases ever since.

In my paper in the Clinical Society's Transactions, page 74, I said: "All the cases referred to above seem to have an important bearing. not only upon the pathology of albuminuria, but also on the treatment of it in certain cases ;" and again at page 76 : "I believe that not only in the cases referred to here, but in many others, the retardation of the glomerular circulation by venous engorgement is the chief factor in the causation of suppression of urine and some forms of albuminuria, and consenuentlv. roliof of tonsinn mav give immediate ease to the patient and restore the function of the kidneyz.

"General experience, 1 thusk, has snown that the vascul ir tension produoed by mechanical venous obstruction or by inflammatory engorgement cannot be relieved permanently by drugs, while it can be rapidly alleviated, and serious consequences avoided, by surgical treatment. Free incision or local bleeding is clearly indicated in such cases, and in all cases of increased vascular tension of a tissue, whether the one or the other method should be employed, depends upon the immediate cause of the tension and the anatomical structure of the organ or part involved." As my case in 1888 was probably the first in which incision of the kidney was recognized as a means of relieving pain and causing the albuminuria to disappear, it may be worth while giving the following short account of the case as recorded in the article in the Clinical Society's Transactions, p. 77.

Sudden' Suppression of Urine: Albuminuria : Renal Colic : Incision if Kidney, followed by Relief of Pain and Disappearance of Albuminuria.

In 1888 , at the Western Infirmary, I saw a man who complained of severe pain in the loins, most severe, however, on the right side. It came on suddenly about two months previously, and at the onset was accompanied by rigors and a sudden diminution in the quantity of urine. I ascertained from his medical attendant, the late Dr. John Moyes, of Largs, that the urine at the beginning of the attack contained albumen, but no tube casts were discovered. Specific gravity 1025 to ro3o, but daily quantity of urea diminished; quantity from $25 \mathrm{oz}$. to $35 \mathrm{oz}$. in twenty-four hours. The patient did not complain of headache, nausea, or vomiting, and no other of the characteristic clinical features nausea, or vomiting, and no other of the characteristic clinical features
of nephritis were present, such as anasarca. effusion into serous cavities, anaemia, or uraemic symptoms.

When seen at the infirmary the urine contained a moderate quantity of albumen but no tube casts, and comparatively little deposit was thrown down on standing. There was a history of the passage of small oxalate of lime calculi and of occasional haematuria. While under observation he had several attacks of distinct renal colic, which led me to the conclusion that he was suffering from renal calculis, and he to the conclusion that he was suffering from renal calcultis, and he was. advised to have an operation performed in the hospital. This he
refused, but consented, after some delay, to have it done in private. On exposing the right kidney by a free incision in the loin the organ was seen to be enlarged, of a dark ohocolate colour, and very tense. On examining the kidney with needles for the detection of a stone free bleeding occurred, and as no calculus could be discovered with the needles I made a free incision into the pelvis in order to explore with the finger. The bleeding was very free, and the wound in the cortex had to be plugged with iodoform gauze. No calculus was found. I felt that I had made either an error in diagnosis or that my search had been Imperfect, and left the case with the bellef that harm rather than good had been done by, the operation; but, to the satisfaction of the pationt and myself, he ceased to suffer any pain, the albuminuria disappeared entirely, and afterwards the patient enjoyed excellent health.

In this case, from the onset of the trouble till the time of the operation, albumen was constantly present and the quantity of urine remained considerably below the normal, but after incision the albumen disappeared and remained absent, and the quantity of the urine increased. I have no doubt that in this case the relief of tension by the incision facilitated the renal circulation.

The conclusions I have come to as regards the efficeincy of incision of the kidney is that it is useful in cases of acute or subacute congestive disorders, such as acute nephritis, congestion associated with haematuria, in cases of anuria from toxic poisoning, or in other instances where the functions of the kidney are suddenly arrested without any evidence of mechanical obstruction to the escape of urine. I am glad to see that more importance is now being attached to surgical intervention in the cases I have referred to. REFERENCE.

1 Cases illustrating increased vascular tension in the kidney as a cause of renal pain. haematuria, and albuminuria, with or without tube casts,
symptoms relieved by surgical treatment. Clinical Society's Trunsactions, vol. XRX, p. 65,1896 .

\section{A FATAL CASE OF SECONDARY PAROTITIS.*}

\section{BY BRENNAN DYBALL, M.B., B.S., F.R.C.S.,}

Anaesthetist to the Royal Deven and Exeter Hospital.

Parotitis, as a sequel to injury or disease of the abdominal or pelvic viscera, is a well known but not very common occurrence. Though it may of ten be a troublesome or even a serious complication, it is as a rule overshadowed by the primary lesion. In the following case, however, the parotitis was of such severity as to be the actual cause of death, and it is therefore, I think, of sufficient interest to be reported:

Onset.

Mr. S. S. M., aged 37 , after two days' slight epigastric pain, was seized with intense abdominal pain about 2 a.m. on November 14 th, rgo2. I saw him about two hours later. He was lying on his back with his knees drawn up, complaining of most intense pain all over the abdomen, but worse in the lower part. The pulse was 120 , and the axillary temperature normal. The abdomen was slightly distended and scarcely moved with respiration, which was of the thoracic type; it was very tender and resistant all over, but not more so at any one spot than another; there was no dullness or local swelling, and repeated inquiries failed to elicit a history of any previous pain in the caecal region. There had been no vomiting or previous attacks of indigestion. The tongue was some what coated, and the bowels had not been opened for two days. Most of the teeth were in a shockingly carious condition. In the absence of localizing signs it was decided to wait a short time. Two hours later the pain was just as severe, but was now localized to the right iliae region, which was very tender and resistent to palpation, while the rest of the abdomen was comparatively free from pain and tenderness. He had passed urine and complained of great pain over the bladder when doing so. The urine was quite normal. The pulse was still 120 , but the temperature had risen to $103^{\circ} \mathrm{F}$.

Operation for Appendicitis.

It was clear that the patient was suffering from a very severe type of appendicitis and operation was advised. Dr. Davy saw him with me a few hours later, agreed with the diagnosis and the necessity for operation, and the patient was removed in an ambulance to the Home Hospital where the operation was performed. Chloroform was. given by Dr. Andrew, and Dr. Solly assisted me. On opening the peritoneal cavity a little faecal-smelling thin pus escaped; there were no adhesions and the appendix was easily found. It was much swollen, gangrenous almost up to its junction with the caecum, half an inch from which a small concretion could be felt. There was no perforation. The meso-appendix was acutely inflamed and nearly half an inch thick. After shutting off the operation area with sponges, the appendix was removed by the usual "coat sleeve" method, the stump invaginated removed by the usual "coat sleeve" method, the stump invaginated
into the caecum and buried by a row of Lembert sutures. About an ounce of offensive pus was removed from the pelvis with sponges, after which the operation area and the pelvis were well irrigated with sterilized water and sponged dry. The wound was then closed, drainage being provided by a large rubber tube with a "wick" of gauze, reaching to the pelvis, and a smaller tube down to the caecum. The patient stood the operation very well, but gave a good deal of trouble to the anaesthetist owing to his tongue persistently falling back. Tongue forceps and a gag had therefore to be freely used, the latter causing some damage to his carious teeth.

After-progress.

As far as the abdominal condition is concerned, the after-progress was most satisfactory. There was no vomiting after the operation, the abdomen quickly became free from pain and distension, and moved well with respiration. The discharge from the drainage tubes was merely a little odourless serum; they were shortened daily and soon dispensed with. The incision healed without any trouble, and the sutures were

* Read before the South-Western Branch of the British Medical Association. 


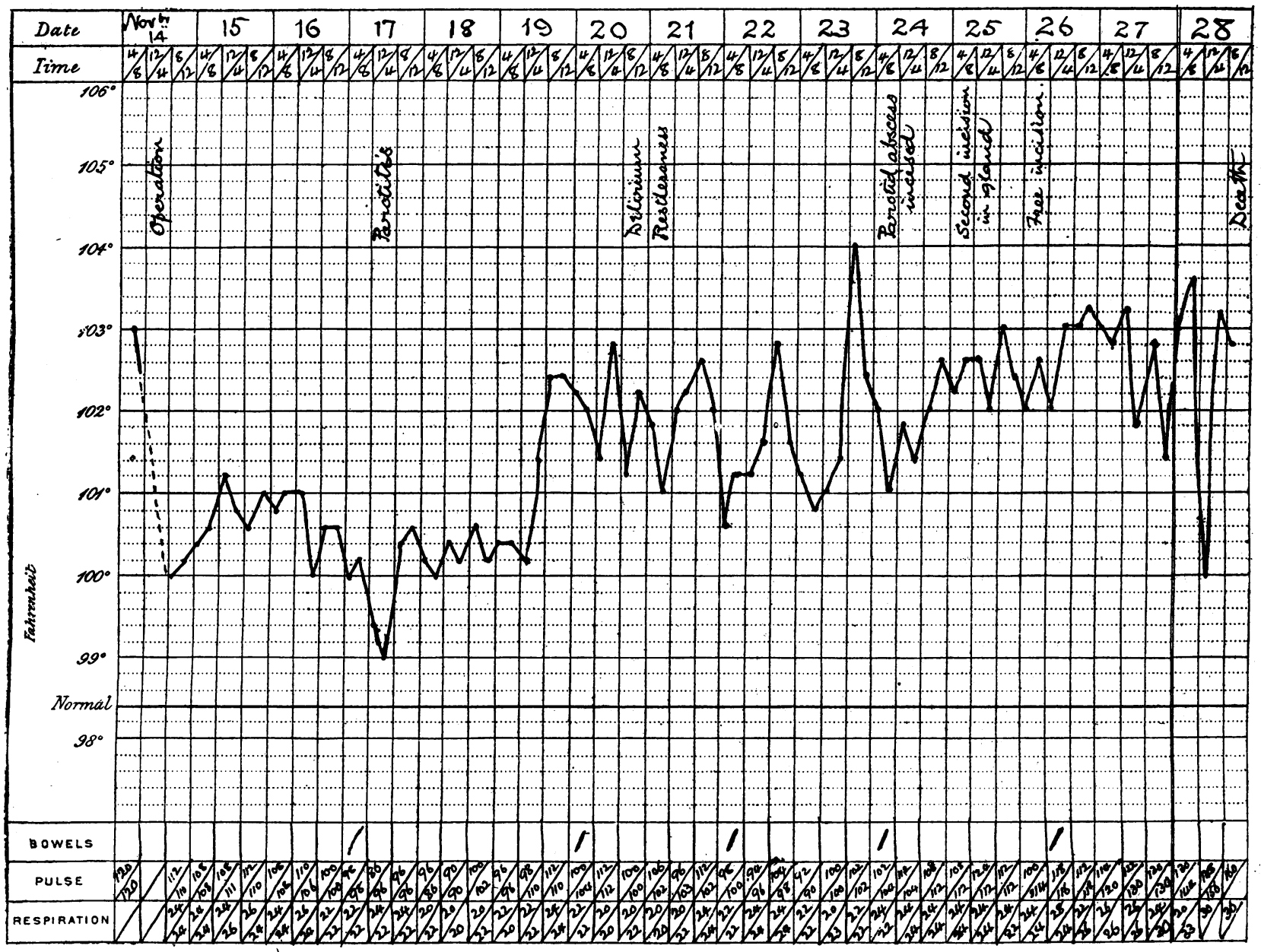

removed on the tenth day. The temperature on the afternoon of the fourth day fell to $99^{\circ} \mathrm{F}$.-in fact, after the first forty-eight hours the abdominal condition gave rise to no anxiety at all.

$$
\text { Onset of Parotitis. }
$$

But, unfortunately, in the reck the state of affairs was very different. on the fourth day the right parotid gland became slightly swollen and tender, and the temperature rose from $99^{\circ} \mathrm{F}$. to roo. $4^{\circ} \mathrm{F}$. A gland beneath the jaw on the same side was also swollen. Hot fomentations of lead lotion were applied, but the parotid bubo increased in size and interfered greatly with feeding owing to the pain on opening the mouth. Two days later the temperature rose to ro2 ${ }^{\circ} \mathrm{F}$. and maintained a high level for the rest of the illness; while on the following day low, muttering delirium, coupled with extreme restlessness set in, and the pulse rose to over roo.

Operation.

Naturally a very keen watch was kept for signs of suppuration, but the swelling kept very hard and brawny, and it was not until the eleventh day that a small spot of softening was detected at the centre. Chloroform was given and the abscess incised by Hilton's method, liberating no more than half a drachm of very thick pus. No improvement in the patient's general condition followed this, and the following day another spot of softening was found at the lower part of the gland and was spot of softening was found at the lower part of the gland and was
incised. The discharge from the first incision was now considerable incised. The discharge from the first incision was now considerable abscess was found to be discharging through the external auditory meatus, and the two cavities were found to be communicating and were laid freely open by an incision joining the other two. The walls of the cavity exposed were in a horrible grey, sloughy, semi-gelatinous state, and the necrosed tissues evidently extended deeply, as a probe passed inwards touched the fronts of the transverse processes and also the base of the skull, but laterally the inflammation was confined fairly to the limits of the gland. Much of the slough was cut away, the cavity was frequently syringed out with a solution of hydrogen peroxide and dressed with hot fomentations of lotio sodae chlorinatae.

After-Treatment

Antistreptococcus serum was injected, brandy and injections of strychnine had been and were freely administered, and the patient held his own extremely well for a time, but on the thirteenth day the heart began to fail and he died on November 28 th, fifteen days after the operation and eleven days after the first appearance of the parotid bubo.

The rarity of parotitis as a cause of death is shown by the fact that only six instances were found in the English American and French literature, and in one of these, reported nearly fifty years ago, death was certainly not due to the parotitis but to the primary abdominal condition. Suppurative parotitis, whether primary or secondary, is not, as a rule, very severe, the pus being confined to the limits of the gland. In the case now related, however, the inflammation was exceptionally virulent, and I can find but very few similar cases in the literature. In one of these the parotid sloughed completely away leaving the masseter bare, but the patient, a boy of ten, recovered. ${ }^{1}$ In another, the inflammation spread beyond the gland with a resulting cellulitis, which extended down to the lower border of the pectoralis major and outwards to the acromion at the death of the patient. ${ }^{2}$ Both of these were cases of primary parotitis. In the third, a case of secondary parotitis after ovariotomy, there was also a consecutive cellulitis of the neck with death of the patient. ${ }^{8}$ In my case it was no doubt owing to a secondary infection of the already-inflamed parotid by septic organisms from the mouth, derived from the broken-down carious teeth, that the inflammation was so virulent. In cancrum oris one sees a similar type of inflammation, also due to the action of buccal organisms.

1 Roth. Gangraena Parotidis. Woch, f.d. ges. Heilk. 1851. 2 Coombs, Med. Press and Circ. r881. ${ }^{3}$ S. Paget, Lancet, i., r886, Case No. 32.

Presentation.-At the conclusion of a course of lectures on home nursing conducted by Mr. H. Norman Barnett, F.R.C.S., at Knock, Belfast, some members of the class presented him with a silver-faced carriage clock as a slight recognition of his services. 